PROCEEDINGS OF THE

AMERICAN MATHEMATICAL SOCIETY

Volume 129, Number 6, Pages 1713-1720

S 0002-9939(00)05709-9

Article electronically published on November 3, 2000

\title{
ASYMPTOTIC BEHAVIOR OF FOURIER TRANSFORMS OF SELF-SIMILAR MEASURES
}

\author{
TIAN-YOU HU
}

(Communicated by Christopher D. Sogge)

\begin{abstract}
Let $\mu$ be a self-similar probability measure on $\mathbb{R}$ satisfying $\mu=$ $\sum_{j=1}^{m} p_{j} \mu \circ F_{j}^{-1}$, where $F_{j}(x)=\rho x+a_{j}, 0<\rho<1, a_{j} \in \mathbb{R}, p_{j}>0$ and $\sum_{j=1}^{m} p_{j}=1$. Let $\hat{\mu}(t)$ be the Fourier transform of $\mu$. A necessary and sufficient condition for $\hat{\mu}(t)$ to approach zero at infinity is given. In particular, if $a_{j}=j$ and $p_{j}=1 / m$ for $j=1, \ldots, m$, then $\lim \sup _{t \rightarrow \infty}|\hat{\mu}(t)|>0$ if and only if $\rho^{-1}$ is a PV-number and $\rho^{-1}$ is not a factor of $m$. This generalizes the corresponding theorem of Erdös and Salem for the case $m=2$.
\end{abstract}

\section{INTRODUCTION}

For $j=1,2, \ldots, m$, let $F_{j}(x)=\rho x+a_{j}$, where $0<\rho<1, a_{j} \in \mathbb{R}$ be a family of equicontractive similitudes and let $p_{1}, p_{2}, \ldots, p_{m}$ be associated probability weights. Then there is a unique self-similar probability measure $\mu$ on $\mathbb{R}$ with compact support satisfying

$$
\mu(A)=\sum_{j=1}^{m} p_{j} \mu\left(F_{j}^{-1}(A)\right)
$$

for all measurable sets $A$.

The measure $\mu$ can also be generated as follows.

Let $X_{0}, X_{1}, \ldots$ be a sequence of i.i.d. random variables each taking real values $a_{1}, a_{2}, \ldots, a_{m}$ with probability $p_{1}, p_{2}, \ldots, p_{m}$ respectively. For $0<\rho<1$ let

$$
S=\sum_{j=0}^{\infty} \rho^{j} X_{j}
$$

and let $\mu_{\rho}$ be the probability measure induced by $S$, i.e., $\mu_{\rho}(A)=\operatorname{Pr} o b\{\omega: S(\omega) \in$ $A\}$. Then it can be verified that this measure also satisfies the above self-similar equation. By uniqueness we obtain $\mu=\mu_{\rho}$.

It is known that $\mu$ is either purely singular or absolutely continuous [JW]. If $0<\rho<1 / m$, then the support of $\mu$ is a set of Cantor type; hence $\mu$ is purely singular. If $1 / m \leq \rho<1$, then the distribution type of $\mu$ depends on the choice of the parameters $a_{1}, a_{2}, \ldots, a_{m}$ and $p_{1}, p_{2}, \ldots, p_{m}$, the determination of which type in general is very difficult.

Received by the editors August 6, 1999 and, in revised form, September 16, 1999.

2000 Mathematics Subject Classification. Primary 42A38; Secondary 28A80.

Key words and phrases. Fourier transform, PV-number, self-similar measure.

(C)2000 American Mathematical Society 
The most fascinating case is when $m=2$ and each $X_{j}$ takes two values \pm 1 with equal probability $1 / 2$. Then $\mu$ is the so-called infinitely convolved Bernoulli measures. If $\rho=1 / 2$, then $\mu$ is a measure proportional to the Lebesgue measure on $[-2,2]$. It was conjectured that $\mu$ ought to be absolutely continuous for $\rho>1 / 2$. However, in 1939 Erdös $\left[\mathrm{E}\right.$ ) showed that if $\rho^{-1}$ is a PV-number and $\rho \neq 1 / 2$ (recall that an algebraic integer $\beta>1$ is called a PV-number if all its conjugate roots have modulus strictly less than one), then the Fourier transform $\hat{\mu}(t)=\int_{-\infty}^{\infty} e^{i t x} d \mu(x)$ does not tend to zero at infinity and thus $\mu$ is purely singular. Salem [S1] showed that for $0<\rho<1$ with $\rho \neq 1 / 2, \hat{\mu}(t) \not \rightarrow 0$ as $t \rightarrow \infty$ only if $\rho^{-1}$ is a PV-number. Since $\mu$ is purely singular for $0<\rho<1 / 2$, the Erdös-Salem theorem gives a large family of singular measures whose Fourier transforms tend to zero at infinity.

In the other direction, Erdös showed that there exists a sequence $\rho_{k} \rightarrow 1$ such that $\mu$ has $k$ derivatives for almost all $\rho \in\left(\rho_{k}, 1\right)$. The result has been generalized recently by Solomyak [So]. He proved that $\hat{\mu}(t)$ is in $L^{2}$ for almost all $1 / 2<\rho$ $<1$ and hence $\mu$ is absolutely continuous with an $L^{2}$ density function. However, the only explicit values of $\rho$ for which $\mu$ is known to be absolutely continuous are $\rho=2^{-1 / n}$, for $n=1,2, \ldots$, and a family of algebraic numbers found by Garsia [G]. On the other hand, all PV-numbers are the only numbers for which $\mu$ is known to be purely singular.

The Fourier transform of the probability measure induced by $\rho^{j} X_{j}$ in (1.1) is $\sum_{k=1}^{m} p_{k} e^{i t a_{k} \rho^{j}}$. Thus the Fourier transform of $\mu$ induced by $S$ is

$$
\hat{\mu}(t)=\prod_{j=0}^{\infty}\left(\sum_{k=1}^{m} p_{k} e^{i t a_{k} \rho^{j}}\right) .
$$

The average asymptotic rate of $\hat{\mu}(t)$ with some restriction on the parameters was studied extensively by Strichartz [St1]-St3, Lau [L1]-L2, Lau and Wang [LW] and Fan and Lau [FL]. In this paper we will give a necessary and sufficient condition for $\hat{\mu}(t)$ to approach zero at infinity. The criterion can be applied to various cases for which a specific set of parameters is given. The detailed proof is given in section 2. In section 3, as an application, we generalize the above Erdös-Salem theorem to $m \geq 2$.

\section{The general theorems}

Let $\mu$ be the probability measure induced by $S$ as defined by (1.1) and let $\hat{\mu}(t)$ be its Fourier transform. Replacing $t$ by $\rho^{-n} t$ in $(1.2)$ we obtain for $n=1,2, \ldots$,

$$
\hat{\mu}\left(\rho^{-n} t\right)=\hat{\mu}(t) \prod_{j=1}^{n}\left(\sum_{k=1}^{m} p_{k} e^{i t a_{k} \rho^{-j}}\right) .
$$

For $j=0, \pm 1, \ldots$, the convex combination $\sum_{k=1}^{m} p_{k} e^{i t a_{k} \rho^{j}}$ lies inside the closed unit disk. We write

$$
d_{j}(t)=1-\left|\sum_{k=1}^{m} p_{k} e^{i t a_{k} \rho^{j}}\right|
$$

for the distance from this convex combination to the circumference of the unit circle. Note that $d_{j}(t)$ depends also on $\rho, a_{1}, a_{2}, \ldots, a_{m}$ and $p_{1}, p_{2}, \ldots, p_{m}$. This notation will be used for the rest of the paper. Furthermore it is easy to check that 
for $j=0, \pm 1, \ldots$,

$$
d_{j}(t) \leq \operatorname{diam}\left\{e^{i t a_{1} \rho^{j}}, e^{i t a_{2} \rho^{j}}, \ldots, e^{i t a_{m} \rho^{j}}\right\} .
$$

Lemma 2.1. Let $\hat{\mu}(t)$ be defined by (1.2). Then $\hat{\mu}(t)=0$ if and only if $\sum_{k=1}^{m} p_{k} e^{i t a_{k} \rho^{j}}=0$ for some $j=0,1, \ldots$

Proof. It suffices to show that if $\sum_{k=1}^{m} p_{k} e^{i t a_{k} \rho^{j}} \neq 0$ for all $j=0,1, \ldots$, then $|\hat{\mu}(t)|>$ 0 .

Using (2.2) and noting that $t a_{k} \rho^{j} \rightarrow 0$ as $j \rightarrow \infty$ we obtain

$$
\begin{aligned}
\sum_{j=0}^{\infty} d_{j}(t) & \leq \sum_{j=0}^{\infty} \operatorname{diam}\left\{e^{i t a_{1} \rho^{j}}, e^{i t a_{2} \rho^{j}}, \ldots, e^{i t a_{m} \rho^{j}}\right\} \\
& \leq 2 \sum_{j=0}^{\infty} \max \left\{\left|t a_{1} \rho^{j}\right|,\left|t a_{2} \rho^{j}\right|, \ldots,\left|t a_{m} \rho^{j}\right|\right\} \\
& <\infty .
\end{aligned}
$$

Since $\sum_{k=1}^{m} p_{k} e^{i t a_{k} \rho^{j}} \neq 0$, we have $1-d_{j}(t)>0$ for all $j$. It follows that

$$
|\hat{\mu}(t)|=\lim _{n \rightarrow \infty} \prod_{j=0}^{n}\left(1-d_{j}(t)\right)>0 .
$$

Q.E.D.

Lemma 2.2. Let $\hat{\mu}(t)$ be defined by (1.2) and let $t \in \mathbb{R}$ with $t \neq 0$ be any point. If either $\sum_{j=1}^{\infty} d_{-j}(t)=\infty$ or $\sum_{k=1}^{m} p_{k} e^{i t a_{k} \rho^{j}}=0$ for some $j=0, \pm 1, \ldots$, then $\lim _{n \rightarrow \infty} \hat{\mu}\left(\rho^{-n} t\right)=0$. Otherwise, $\lim _{n \rightarrow \infty}\left|\hat{\mu}\left(\rho^{-n} t\right)\right|>0$.

Proof. If $\sum_{k=1}^{m} p_{k} e^{i t a_{k} \rho^{j}}=0$ for some $j=0, \pm 1, \ldots$, then by $(2.1) \hat{\mu}\left(\rho^{-n} t\right)=0$ for all large $n$, and we have trivially $\lim _{n \rightarrow \infty} \hat{\mu}\left(\rho^{-n} t\right)=0$.

Suppose that $\sum_{k=1}^{m} p_{k} e^{i t a_{k} \rho^{j}} \neq 0$ for all $j=0, \pm 1, \ldots$. Then $|\hat{\mu}(t)|>0$ by Lemma 2.1 and $1-d_{-j}(t)>0$ for all $j=1,2, \ldots$. This, along with (2.1), implies that

$$
\lim _{n \rightarrow \infty}\left|\hat{\mu}\left(\rho^{-n} t\right)\right|=|\hat{\mu}(t)| \lim _{n \rightarrow \infty} \prod_{j=1}^{n}\left(1-d_{-j}(t)\right)>0
$$

if and only if $\sum_{j=1}^{\infty} d_{-j}(t)<\infty$. Q.E.D.

Theorem 2.3. Let $\hat{\mu}(t)$ be defined by (1.2). If for every $t \in \mathbb{R}$ with $t \neq 0$ either $\sum_{j=1}^{\infty} d_{-j}(t)=\infty$ or $\sum_{k=1}^{m} p_{k} e^{i t a_{k} \rho^{j}}=0$ for some $j=0, \pm 1, \ldots$, then $\lim _{x \rightarrow \infty} \hat{\mu}(x)$ $=0$. Otherwise, $\lim \sup _{x \rightarrow \infty}|\hat{\mu}(x)|>0$ and hence $\mu$ is purely singular.

Proof. In view of Lemma 2.2 it suffices to show that if $\lim _{n \rightarrow \infty} \hat{\mu}\left(\rho^{-n} t\right)=0$ for every $t \in[\rho \pi, \pi]$, then $\lim _{x \rightarrow \infty} \hat{\mu}(x)=0$. We will show this by contradiction. Suppose that there is a sequence $t_{n} \rightarrow \infty$ and an integer $n_{0}$ such that

$$
\left|\hat{\mu}\left(t_{n}\right)\right| \geq \delta>0 \text { for all } n \geq n_{0} .
$$

Let $k_{n}, n=1,2, \ldots$, be a sequence of positive integers such that $\rho^{k_{n}} t_{n} \in[\rho \pi, \pi]$. Without loss of generality, assume that $\lim _{n \rightarrow \infty} \rho^{k_{n}} t_{n}=t \in[\rho \pi, \pi]$. By assumption, $\lim _{n \rightarrow \infty} \hat{\mu}\left(\rho^{-n} t\right)=0$. Hence for any $\varepsilon>0$ there exists an integer, which may also be taken to be $n_{0}$, such that for all $n \geq n_{0}$

$$
\left|\hat{\mu}\left(\rho^{-n} t\right)\right|<\varepsilon .
$$


Note that for this fixed $n_{0}, \hat{\mu}\left(\rho^{-n_{0}} x\right)$ is a continuous function of $x$. Using

$$
\lim _{n \rightarrow \infty} \rho^{k_{n}} t_{n}=t
$$

we have for all large $n$

$$
\left|\hat{\mu}\left(\rho^{k_{n}-n_{0}} t_{n}\right)-\hat{\mu}\left(\rho^{-n_{0}} t\right)\right|<\varepsilon .
$$

By (2.1) we have $\left|\hat{\mu}\left(\rho^{-n} t\right)\right| \leq|\hat{\mu}(t)|$ or equivalently $|\hat{\mu}(t)| \leq\left|\hat{\mu}\left(\rho^{n} t\right)\right|$ for every $t$ and for every positive integer $n$. It follows from (2.4) and (2.5) that for all large $n$

$$
\left|\hat{\mu}\left(t_{n}\right)\right| \leq\left|\hat{\mu}\left(\rho^{k_{n}-n_{0}} t_{n}\right)\right|<\left|\hat{\mu}\left(\rho^{-n_{0}} t\right)\right|+\varepsilon<2 \varepsilon .
$$

This contradicts (2.3) if $\varepsilon$ is chosen to be less than $\delta / 3$. Q.E.D.

Next, we give a condition so that $\lim \sup _{x \rightarrow \infty}|\hat{\mu}(x)|>0$ if $\rho^{-1}$ is a PV-number.

For any real number $a$, we denote by $\|a\|$ the distance between $a$ and the nearest integer. Thus $\|a\|=\min |a-n|, n=0, \pm 1, \ldots$ If $k$ is the integer nearest to $a$, we write $a=k+\langle a\rangle$ so that $\|a\|=|\langle a\rangle| \leq 1 / 2$.

It is known that $\beta>1$ is a $\mathrm{PV}$-number if and only if there exists a real number $t \neq 0$ such that $\sum_{j=1}^{\infty}\left\|t \beta^{j}\right\|^{2}<\infty$. It is also known that for any given PV-number $\beta$ the set

$$
E(\beta)=\left\{t: \sum_{j=1}^{\infty}\left\|t \beta^{j}\right\|^{2}<\infty\right\}
$$

is countable [S2, BDGPS].

If $\beta>1$ is a $\mathrm{PV}$-number, then $\left\|\beta^{j}\right\|$ tends to zero geometrically, and so does $\left\|k \beta^{j}\right\|$ for any fixed integer $k$; hence $k \in E(\beta)$ for every integer $k$. Furthermore, if $t=k \beta^{j_{0}}$ for some integer $k$ and for some $j_{0}=0, \pm 1, \ldots$, then $\left\|t \beta^{j}\right\|=\left\|k \beta^{j+j_{0}}\right\|$ tends to zero geometrically. It follows that $k \beta^{j_{0}} \in E(\beta)$ for arbitrary integers $k, j_{0}$. In Lemma 3.1 we will show that if $\beta \geq 2$ is an integer, then $E(\beta)$ consists precisely these numbers.

Theorem 2.4. Let $\rho^{-1}$ be a PV-number and let $\hat{\mu}(t)$ be defined by (1.2). If $a_{k} \in$ $E\left(\rho^{-1}\right)$ for $k=1, \ldots, m$ and if there exists a positive integer $N$ such that

$$
\sum_{k=1}^{m} p_{k} e^{i 2 N \pi a_{k} \rho^{j}} \neq 0
$$

for all $j=0, \pm 1, \ldots$, then $\lim _{n \rightarrow \infty}\left|\hat{\mu}\left(\rho^{-n} 2 N \pi\right)\right|>0$. In particular, $\mu$ is purely singular.

Proof. Using Lemma 2.2 with $t=2 N \pi$ we need only to show that $\sum_{j=1}^{\infty} d_{-j}(2 N \pi)$ $<\infty$, where $d_{-j}(2 N \pi)=1-\left|\sum_{k=1}^{m} p_{k} e^{i 2 N \pi a_{k} \rho^{-j}}\right|$. Since $a_{k} \in E\left(\rho^{-1}\right)$ for $k=$ $1, \ldots, m$, we have

$$
\sum_{j=1}^{\infty} \max \left\{\left\|a_{1} \rho^{-j}\right\|^{2},\left\|a_{2} \rho^{-j}\right\|^{2}, \ldots,\left\|a_{m} \rho^{-j}\right\|^{2}\right\}<\infty
$$


In particular, $\max \left\{\left\|a_{1} \rho^{-j}\right\|,\left\|a_{2} \rho^{-j}\right\|, \ldots,\left\|a_{m} \rho^{-j}\right\|\right\} \rightarrow 0$, as $j \rightarrow \infty$. We have

$$
\begin{aligned}
\left|\sum_{k=1}^{m} p_{k} e^{i 2 N \pi a_{k} \rho^{-j}}\right| & \geq \operatorname{Re}\left(\sum_{k=1}^{m} p_{k} e^{i 2 N \pi a_{k} \rho^{-j}}\right) \\
& \geq \min _{1 \leq k \leq m}\left\{\operatorname{Re}\left(e^{i 2 N \pi a_{k} \rho^{-j}}\right)\right\} \\
& =\min _{1 \leq k \leq m}\left\{\cos 2 N \pi\left\|a_{k} \rho^{-j}\right\|\right\} \\
& =\cos \theta_{j} \\
& \geq 1-\frac{\theta_{j}^{2}}{2},
\end{aligned}
$$

where $\theta_{j}=2 N \pi \max \left\{\left\|a_{1} \rho^{-j}\right\|,\left\|a_{2} \rho^{-j}\right\|, \ldots,\left\|a_{m} \rho^{-j}\right\|\right\}$. Hence for large $j$ we have $d_{-j}(2 N \pi) \leq \theta_{j}^{2} / 2$ and

$$
\sum_{j=1}^{\infty} d_{-j}(2 N \pi) \leq 2 N^{2} \pi^{2} \sum_{j=1}^{\infty} \max \left\{\left\|a_{1} \rho^{-j}\right\|^{2},\left\|a_{2} \rho^{-j}\right\|^{2}, \ldots,\left\|a_{m} \rho^{-j}\right\|^{2}\right\}<\infty .
$$

Q.E.D.

\section{A THEOREM of ERdös AND SAlEM}

In this section we will generalize a theorem of Erdös and Salem on the asymptotic behavior of Fourier transforms of infinitely convolved Bernoulli measures.

Lemma 3.1. Let $n \geq 2$ be any given integer and let $t \in \mathbb{R}$ with $t \neq 0$. If $t=k / n^{j_{0}}$ for some integer $k$ and for some $j_{0}=0,1, \ldots$, then $\left\langle t n^{j}\right\rangle=0$ for all $j \geq j_{0}$. Otherwise, $\lim \sup _{j \rightarrow \infty}\left\|t n^{j}\right\|>0$.

Proof. Write $t n^{j}=k_{j}+\left\langle t n^{j}\right\rangle$, where $k_{j}$ is the integer nearest to $t n^{j}$. Then

$$
t n^{j+1}=n k_{j}+n\left\langle t n^{j}\right\rangle \text {. }
$$

If $0<\left|\left\langle t n^{j}\right\rangle\right|<\frac{1}{2 n}$, then $0<n\left|\left\langle t n^{j}\right\rangle\right|<1 / 2$. Hence $\left|\left\langle t n^{j}\right\rangle\right|<n\left|\left\langle t n^{j}\right\rangle\right|=\left|\left\langle t n^{j+1}\right\rangle\right|$. It follows that the union of the two intervals $\left(-\frac{1}{2 n}, 0\right) \cup\left(0, \frac{1}{2 n}\right)$ is a repelling region for $\left\langle t n^{j}\right\rangle$. Therefore $\lim \sup _{j \rightarrow \infty}\left\|t n^{j}\right\|>0$ unless $\left\langle t n^{j}\right\rangle=0$ for all $j \geq j_{0}$ and for some $j_{0}$, which is equivalent to $t n^{j_{0}}=k$ for some integer $k$ and for some $j_{0}=0,1, \ldots$ Q.E.D.

Theorem 3.2 (A generalized theorem of Erdös-Salem). Let $S$ be defined by (1.1) with each $X_{j}$ taking $m$ values $0,1, \ldots, m-1$ with equal probability $1 / m$. Let $\mu$ be the probability measure induced by $S$ and let $\hat{\mu}$ be its Fourier transform. If $\rho^{-1}$ is a $P V$-number and $\rho^{-1}$ is not a factor of $m$, then $\lim \sup _{t \rightarrow \infty}|\hat{\mu}(t)|>0$. Otherwise, $\lim _{t \rightarrow \infty} \hat{\mu}(t)=0$.

Proof. Suppose that $\rho^{-1}$ is a PV-number which is not a factor of $m$. Since all integers are contained in $E\left(\rho^{-1}\right)$, using Theorem 2.4 with $N=1$ we need to verify that

$$
\sum_{k=0}^{m-1} e^{i 2 k \pi \rho^{j}} \neq 0 \text { for all } j=0, \pm 1, \ldots
$$

We will show that if $\sum_{k=0}^{m-1} e^{i 2 k \pi \rho^{j}}=0$ for some $j$ and if $\rho^{-1}$ is a PV-number, then it must be a factor of $m$. Clearly $\sum_{k=0}^{m-1} e^{i 2 k \pi \rho^{j}}=0$ implies that $e^{i 2 \pi \rho^{j}} \neq 1$; otherwise 
$\sum_{k=0}^{m-1} e^{i 2 k \pi \rho^{j}}=m \neq 0$. Note that $\left(1-e^{i 2 \pi \rho^{j}}\right) \sum_{k=0}^{m-1} e^{i 2 k \pi \rho^{j}}=1-e^{i 2 m \pi \rho^{j}}$. Hence $\sum_{k=0}^{m-1} e^{i 2 k \pi \rho^{j}}=0$ for some $j$ if and only if $e^{i 2 \pi \rho^{j}}$ is a $m$ th root of the unity, i.e., $\left\langle\rho^{j}\right\rangle=k / m$ for some positive integer $j$ and for some $k=0,1, \ldots, m-1$. Since the only rational $\mathrm{PV}$-numbers are integers greater than or equal to 2 , it implies that $\rho^{j}=k / m$ must be a reciprocal of an integer. Hence $\rho^{-j}$ is a factor of $m$ and so is $\rho^{-1}$. Theorem 2.4 thus shows $\lim \sup _{t \rightarrow \infty}|\hat{\mu}(t)|>0$.

Next, suppose that $\rho^{-1}$ is not a PV-number. Then $\sum_{j=1}^{\infty}\left\|t \rho^{-j}\right\|^{2}=\infty$ for every $t \in \mathbb{R}$ with $t \neq 0$.

Case 1. $m$ is odd. For simplicity let $X_{j}$ take $m$ values in

$$
A= \pm 2 \pi\{0,1, \ldots,(m-1) / 2\}
$$

with equal probability $1 / m$.

Fix any $t \in \mathbb{R}$ with $t \neq 0$. Since $\rho^{-1}$ is not an integer, then $\left\langle t \rho^{-j}\right\rangle=0$ occurs for at most one index $j$. Without loss of generality, assume that $\left\langle t \rho^{-j}\right\rangle \neq 0$ for all $j$. Using the formula $\frac{1}{2}+\cos x+\cos 2 x+\cdots+\cos n x=\frac{\sin \left(n+\frac{1}{2}\right) x}{2 \sin \frac{x}{2}}$ for $x \neq 2 k \pi$ we have

$$
\begin{aligned}
\left|\frac{1}{m} \sum_{a \in A} e^{i a t \rho^{-j}}\right| & =\frac{1}{m}\left|1+2 \sum_{k=1}^{(m-1) / 2} \cos 2 k \pi t \rho^{-j}\right| \\
& =\frac{1}{m}\left|\frac{\sin \left(\frac{m-1}{2}+\frac{1}{2}\right) 2 \pi t \rho^{-j}}{\sin \pi t \rho^{-j}}\right| \\
& =\frac{1}{m} \frac{\left|\sin m \pi\left\langle t \rho^{-j}\right\rangle\right|}{\sin \pi\left\langle t \rho^{-j}\right\rangle} .
\end{aligned}
$$

If $\left\langle t \rho^{-j}\right\rangle \geq \frac{1}{2 m}$, then

$$
(3.1) \leq \frac{1}{m \sin (\pi / 2 m)}
$$

Consider the Taylor expansion $\frac{\sin m x}{\sin x}=m-\frac{\left(m^{3}-m\right) x^{2}}{3 !}+\left(\frac{m^{5}-m}{5 !}-\frac{m^{3}-m}{3 ! 3 !}\right) x^{4}-\cdots$. If $\left\langle t \rho^{-j}\right\rangle<\frac{1}{2 m}$, then

$$
\begin{aligned}
(3.1) & =1-\frac{m^{2}-1}{3 !} \pi^{2}\left\langle t \rho^{-j}\right\rangle^{2}+\left(\frac{m^{4}-1}{5 !}-\frac{m^{2}-1}{3 ! 3 !}\right) \pi^{4}\left\langle t \rho^{-j}\right\rangle^{4}-\cdots \\
& \leq 1-c_{m}\left\langle t \rho^{-j}\right\rangle^{2}
\end{aligned}
$$

where $c_{m}>\frac{m^{2}-1}{3 !} \pi^{2}$ is some constant depending only on $m$ such that $1-c_{m}\left\langle t \rho^{-j}\right\rangle^{2}$ $>0$. Using the hypothesis $\sum_{j=1}^{\infty}\left\|t \rho^{-j}\right\|^{2}=\infty$ we obtain

$$
\begin{aligned}
\sum_{j=1}^{\infty} d_{-j}(t) & =\sum_{j=1}^{\infty}\left(1-\left|\frac{1}{m} \sum_{a \in A} e^{i a t \rho^{-j}}\right|\right) \\
& \geq \sum_{\left\langle t \rho^{-j}\right\rangle<\frac{1}{2 m}} c_{m}\left\langle t \rho^{-j}\right\rangle^{2}+\sum_{\left\langle t \rho^{-j}\right\rangle \geq \frac{1}{2 m}}\left(1-\frac{1}{m \sin (\pi / 2 m)}\right) \\
& =\infty .
\end{aligned}
$$

Hence $\lim _{t \rightarrow \infty} \hat{\mu}(t)=0$ by Theorem 2.3 . 
Case 2. $m$ is even. For simplicity we let $X_{j}$ take $m$ values in

$$
A= \pm 2 \pi\{1,3,5, \ldots, m-1\}
$$

with equal probability $1 / m$.

Fix any $t \in \mathbb{R}$ with $t \neq 0$. Using the formula $\cos x+\cos 3 x+\cdots+\cos (2 n-1) x=$ $\frac{\sin n x \cos n x}{\sin x}$ we have

$$
\begin{aligned}
\left|\frac{1}{m} \sum_{a \in A} e^{i a t \rho^{-j}}\right| & =\frac{2}{m}\left|\sum_{k=1}^{m / 2} \cos 2(2 k-1) \pi t \rho^{-j}\right| \\
& =\frac{2}{m}\left|\frac{\sin m \pi t \rho^{-j} \cos m \pi t \rho^{-j}}{\sin 2 \pi t \rho^{-j}}\right| \\
& =\frac{1}{m}\left|\frac{\sin 2 m \pi\left\langle t \rho^{-j}\right\rangle}{\sin 2 \pi\left\langle t \rho^{-j}\right\rangle}\right|
\end{aligned}
$$

which is similar to (3.1). Applying the same argument we obtain $\lim _{t \rightarrow \infty} \hat{\mu}(t)=0$.

Finally, we will show that if $\rho^{-1}$ is a positive integer which is a factor of $m$, then $\lim _{t \rightarrow \infty} \hat{\mu}(t)=0$. We let $X_{j}$ take $m$ values in $2 \pi\{0,1,2, \ldots,(m-1)\}$ with equal probability $1 / \mathrm{m}$. By Theorem 2.3 it suffices to show that for every $t \in \mathbb{R}$ with $t \neq 0$ either $\sum_{j=1}^{\infty} d_{-j}(t)=\infty$ or $\sum_{k=0}^{m-1} e^{i 2 k \pi t \rho^{j}}=0$ for some integer $j=0, \pm 1, \ldots$.

Suppose that $t=k_{0} \rho^{j_{0}}$ for some integer $k_{0}$ and for some $j_{0}=0, \pm 1, \ldots$. Without loss of generality, assume that $k_{0} \rho$ is not an integer. Otherwise, we write $t=k_{1} \rho^{j_{0-1}}$, where $k_{1}=k_{0} \rho$. Let $j=1-j_{0}$. Then $t \rho^{j}=k_{0} \rho$ is not an integer and hence $e^{i 2 \pi t \rho^{j}} \neq 1$. Since $\rho^{-1}$ is a factor of $m$, i.e., $m \rho$ is an integer; hence $e^{i 2 m \pi t \rho^{j}}=$ $e^{i 2 m \pi k_{0} \rho}=1$. It follows that

$$
\sum_{k=0}^{m-1} e^{i 2 k \pi t \rho^{j}}=\frac{1-e^{i 2 m \pi t \rho^{j}}}{1-e^{i 2 \pi t \rho^{j}}}=0 .
$$

If $t \neq k_{0} \rho^{j_{0}}$ for any integer $k_{0}$ and for any $j_{0}=0,1, \ldots$, then by Lemma $3.1\left\|t \rho^{-j}\right\|$ does not tend to zero as $j \rightarrow \infty$. This certainly implies that $\sum_{j=1}^{\infty}\left\|t \rho^{-j}\right\|^{2}=\infty$. So $\sum_{j=1}^{\infty} d_{-j}(t)=\infty$ by above argument and hence $\lim _{t \rightarrow \infty} \hat{\mu}(t)=0$. Q.E.D.

\section{ACKNOWLEDGMENT}

The author would like to thank Professor R. S. Strichartz for pointing out an error in the first draft of the manuscript.

\section{REFERENCES}

[BDGPS] M. Bertin, A. Decomps-Guilloux, M. Grandet-Hugot, M. Pathiaux-Delefosse and J. Schreiber, Pisot and Salem Numbers, Birkhäuser-Verlag, Basel, 1992. MR 93k:11095

[E] P. Erdös, On a family of symmetric Bernoulli convolutions, Amer. J. Math. 61 (1939), 974-975. MR 1:52a

[FL] A. Fan and K. Lau, Asymptotic behavior of multiperiodic functions $G(x)=$ $\prod_{j=0}^{\infty} g\left(x / 2^{n}\right)$, J. Four. Anal. and Appl., 4 (1998), 130-150. MR 99j:41054

[G] A. M. Garsia, Arithmetic properties of Bernoulli convolutions, Trans. Amer. Math. Soc. 102 (1962), 409-432. MR 25:1409

[JW] B. Jessen and A. Wintner, Distribution functions and the Riemann zeta function, Trans. Amer. Math. Soc. 38 (1935), 48-88.

[L1] K. Lau, Fractal measures and mean p-variations, J. Funct. Anal. 108 No.2 (1992), 427-457. MR 93g:28007 
[L2] K. Lau, Dimension of a family of singular Bernoulli convolutions, J. Funct. Anal. 116 No.2 (1993), 335-358. MR 95h:28013

[LW] K. Lau and J. Wang, Mean quadratic variations and Fourier asymptotics of self-similar measures, Monatsch Math., 115 (1993), 99-132. MR 94g:42018

[S1] R. Salem, A remarkable class of algebraic integers. Proof of a conjecture of Vijayaraghavan, Duke Math. J. 11 (1944), 103-108. MR 5:254a

[S2] R. Salem, Algebraic Numbers and Fourier Analysis, Heath, Boston (1963). MR 28:1169

[So] B. Solomyak, On the Random Series $\sum \pm \lambda^{n}$ (an Erdös problem), Annals of Math. 142 (1995), 611-625. MR 97d:11125

[St1] R. Strichartz, Fourier asymptotics of fractal measures, J. Funct. Anal. 89 (1990), 154187. MR 91m:42015

[St2] R. Strichartz, Self-Similar measures and their Fourier transforms I, Indiana Univ. Math. J., 39 (1990), 797-817. MR 92k:42015

[St3] R. Strichartz, Self-Similar measures and their Fourier transforms II, Trans. Amer. Math. Soc., 336 (1993), 335-361. MR 93e:42023

Department of Mathematics, University of Wisconsin-Green Bay, Green Bay, WisCONSIN 54311

E-mail address: hut@uwgb.edu 